\title{
Efecto de la calidad de un curso sobre manejo poscosecha en la motivación, aprendizaje y rendimiento estudiantil en la Universidad Estatal a Distancia de Costa Rica
}

\author{
Carmen Andrés Jiménez \\ Universidad Estatal a Distancia (UNED), 474-2050 Mercedes de Montes de Oca, San José-Costa Rica; candres@uned.ac.cr
}

Recibido 27-III-2012 Corregido 11-VI-2012 Aceptado 12-VII-2012

\begin{abstract}
Effect of the quality of a postharvest handling course in the motivation, learning process and performance of students at the Distance State University of Costa Rica. Performance, motivation and knowledge of the students are the three central axes in which the Agroindustry chair has been working since the year 2012 to improve the quality of its courses and take care of the students in a better way. My hypothesis was that if each student situation is taken into account, and the learning process is not based only on a final grade, there would be better motivation, knowledge and performance. The Postharvest handling course (first term of 2012) at UNED was investigated through: a) an evaluation of the level of the students' motivation with a survey and inquiry about their specific situation and b) an analysis of their performance and knowledge level through the judgment of two evaluation instruments: Forum 1 and the first exam. The students do not respond in a positive way if the alternative evaluation activities lack a percentage in the final grade, or they do not get enough feedback about their evaluations; the prefer to have more presential tutorials. Knowledge is negatively correlated with the final grade obtained by the students in Forum 1, but positively related to the first exam. The knowledge of the students is not completely measured with a final grade and individual situations must be taken into account to increase motivation, performance and knowledge. I recommend the inclusion of alternative evaluation activities in the Agroindustry chair without leaving behind the specific situations of each student.
\end{abstract}

\section{KEY WORDS}

Evaluation, postharvest, performance, motivation, knowledge, student, teacher.

\section{RESUMEN}

En la UNED de Costa Rica, el rendimiento, motivación y aprendizaje de los estudiantes son los tres ejes centrales en los que la Cátedra de Agroindustria se está enfocando a partir del año 2012 para mejorar la calidad de los cursos y dar una mejor atención a los estudiantes. La hipótesis que la cátedra plantea es que si se toma en cuenta la situación de cada estudiante y el proceso de aprendizaje no se basa solamente en una nota final, mayor será su motivación, rendimiento y aprendizaje. Se trabajó el curso Manejo poscosecha I, impartido en el I cuatrimestre del 2012, realizando a) una evaluación del nivel de motivación de los estudiantes a través de una encuesta y la indagación de su situación específica y b) un análisis de su rendimiento y nivel de aprendizaje a través del estudio de dos instrumentos de evaluación: foro y examen. Se encontró que los estudiantes no responden de manera positiva a actividades de evaluación alternativa si no tienen porcentaje en la nota final, que no reciben suficiente retroalimentación sobre sus evaluaciones y que prefieren tener más tutorías presenciales. Se encontró también que el nivel de aprendizaje no está relacionado positivamente con el rendimiento obtenido en la nota final del Foro 1, mientras que sí está relacionado en el primer examen ordinario. Se concluye que el aprendizaje de los estudiantes no se mide por completo con una nota final y la situación específica de cada estudiante debe considerarse para mejorar su motivación, aprendizaje y rendimiento. Se recomienda introducir cada vez más la evaluación alternativa en los cursos de la Cátedra de Agroindustria, sin dejar de lado las situaciones específicas de cada estudiante.

\section{PALABRAS CLAVE}

Evaluación, poscosecha, rendimiento, motivación, aprendizaje, estudiante, docente.
La Cátedra de Agroindustria de la Escuela de Ciencias Exactas y Naturales de la UNED la da servicios a las carreras de Ingeniería Agroindustrial, Ingeniería Agronómica y Administración de empresas agropecuarias. Desde al año 2009, la cátedra empezó la migración de todos sus cursos en línea (Andrés, 2010), completándola a finales del año 2010. A partir de este cambio, la cátedra empezó a realizar investigaciones sobre los que considera los tres ejes que sustentan la calidad de sus cursos: motivación, aprendizaje y rendimiento. 
La razón por la cual la cátedra está tan preocupada por investigar estos temas, es porque se realizó un estudio (en prensa) sobre el curso "Control de Calidad" a finales del año 2011, en el que los estudiantes expresaron estar desmotivados, primordialmente por percibir que el curso no constaba con una metodología clara de enseñanza, que los temas de estudio no estaban lo suficientemente claros y no recibir un seguimiento más constante por parte de los tutores (Andrés, 2012).

A raíz de lo anterior, la Cátedra planteó la siguiente hipótesis sobre cómo mejorar los tres ejes que sustentan la calidad de sus cursos: si la cátedra se preocupa por indagar cómo trabaja cada estudiante en relación a la plataforma virtual y la periodicidad con la cual puede ingresar a la misma y si mejora la calidad de la metodología que utiliza para la evaluación de los aprendizajes, no solamente con una nota final, logrará un aumento en el rendimiento, aprendizaje y motivación de los estudiantes.

A partir de esta hipótesis, estudiaremos la literatura que sustenta cada uno de los ejes por separado, comenzando por la motivación.

La motivación es un eje relevante para el éxito de los cursos de la cátedra, ya que sin ella, no es fácil que los estudiantes logren un aprendizaje significativo ni un rendimiento importante.

La cátedra ha visto como los estudiantes y las estudiantes se desmotivan por diferentes razones y por lo tanto se frustran. Al respecto, Borges, 2005 dice "las consecuencias de la frustración del estudiante pueden ser gravosas para todos los agentes implicados en la educación en línea: estudiantes, docentes e instituciones" (Borges, 2005, p. 2).

En el curso Manejo poscosecha l, se está trabajando el concepto de metacognición y autorregulación, motivando a los estudiantes a que comprendan que si su aprendizaje es mayor, su rendimiento también lo será. Se trabaja evaluación tanto formativa (retroalimentación en la tarea, foros, informe de gira) como sumativa (dos examenes ordinarios, dos foros académicos, una tarea y un informe de gira). Gargallo et al., 2009, comentan que a pesar de que las estrategias de aprendizaje se han conceptualizado desde hace muchos años bajo los temas de cognición y metacognición, últimamente también se han enriquecido incluyendo elementos afectivo-motivacionales y de apoyo.

Con respecto a lo anterior, Suárez \& Fernández, 2011, señalan que así como un estudiante puede gestionar su cognición y conducta, también puede gestionar su motivación, lo cual supone una mayor implicación en el proceso de aprendizaje, un mayor nivel de esfuerzo y mejor rendimiento (Suárez \& Fernández, 2011, p. 369).
El segundo eje importante para la Cátedra es el aprendizaje. Se ha comprobado que la mejor manera de aprender es sentir lo que se está haciendo como propio. Al respecto, Peña et al., 2002, mencionan que es importante que el estudiante pueda aprender según sus preferencias, en un entorno amigable, y lo más cercano posible a su estilo de aprendizaje (Peña et al., 2002, p. 1).

El aprendizaje no solamente tiene que ver con el estudiante y la estudiante, sino también con el docente y la docente. Los tutores y las tutoras pueden cometer errores que ponen en peligro el aprendizaje de los y las estudiantes. Entre estos errores, Borges, 2005 señala los siguientes: no dar respuesta inmediata a los estudiantes, tener una presencia esporádica o nula en el aula virtual, no ser claro en sus indicaciones, ser muy rígido, sobrecargar al estudiante y no mostrar cercanía.

Asimismo, la Universidad debe ofrecer una ayuda técnica que sea satisfactoria y accesible al estudiante, debe capacitar de manera correcta al docente en línea y al estudiante y debe tener en cuenta la situación del estudiante (Borges, 2005). Todo esto para que el aprendizaje sea significativo y les sirva para su profesión en el futuro, que es lo que más cuenta.

Un factor adicional a tomar en cuenta para aumentar el nivel de aprendizaje de los y las estudiantes, es la variabilidad de la población estudiantil en nuestra universidad. Según el documento para la elaboración de cursos en línea de la Universidad Estatal a Distancia (UNED), la posibilidad de utilizar una tecnología que facilite el acceso a los recursos educativos en cualquier tiempo y lugar, ha hecho posible la aparición de nuevos públicos, antiguamente marginados por un sistema educativo rígido y presencial (PAL-PACE-UNED, 2010). Es por eso que no debemos perder de vista que la población estudiantil que manejamos es muy diversa y no todas las personas pueden accesar de la misma manera a los recursos tecnológicos.

Con respecto a este tema, la Cátedra de Agroindustria ha desarrollado diferentes sistemas mediante los cuales incluye a las personas con difícil acceso a la tecnología dentro de sus cursos, sin hacerlos sentir excluidos o desmotivados.

Por último, el diseño del entorno virtual en el que se imparten los cursos también es crucial para el aprendizaje. No es lo mismo un curso a distancia tradicional (con unidad didáctica, tareas y exámenes) a un curso virtual, en el que se cuenta con herramientas tecnológicas que pueden hacer la experiencia de enseñanza más dinámica y hacer que el y la estudiante aprenda mucho más (Pérez, 2012).

El tercer eje que sustenta la calidad de la Cátedra es el rendimiento. Un buen rendimiento va unido a una buena evaluación. Si no se evalúa, no hay retroalimentación, 
no se conocen los resultados, no se identifican los puntos débiles, los puntos fuertes, los ajustes ni las conexiones (Sánchez-Torres, 2006).

Para determinar si se está realizando una buena evaluación de los aprendizajes, existen varias teorías, pero los indicadores son aún incipientes. Sin embargo, Cardona \& Sánchez, 2010, recomiendan que los indicadores se basen en 5 aspectos: institución (soporte tecnológico por parte de la institución), pedagogía (materiales, metodología, asesoría del tutor, retroalimentaciones), tecnología (accesibilidad, habilidades del estudiante), contexto (social, económico, cultural, político), servicios (satisfacción del estudiante y motivación).

Agregan los autores citados, que la evaluación alternativa ha logrado ayudar al profesorado a maximizar el aprendizaje de los estudiantes. La evaluación no es solamente una calificación, sino una reflexión del docente, un control de calidad para tomar decisiones y mejorar el proceso de aprendizaje.

Para que una evaluación sea orientada al aprendizaje de los y las estudiantes, debe contemplar los siguientes aspectos: (a) Plantear las tareas de evaluación como tareas de aprendizaje, (b) involucrar a los estudiantes en la evaluación y (c) ofrecer los resultados de la evaluación a modo de retroalimentación (Cardona \& Sánchez, 2010).

Con base en lo mencionado anteriormente sobre los tres ejes que sustentan la calidad de la Cátedra de Agroindustria, el objetivo principal de esta investigación es evaluar el efecto que tuvo la calidad del curso Manejo poscosecha I en la motivación, aprendizaje y rendimiento de los estudiantes que lo llevaron durante el I cuatrimestre del año 2012 en la UNED.

Cabe aclarar que la presente investigación es un estudio parcial, de tipo cuantitativo, realizado a mitad de periodo lectivo, por lo que los resultados reflejan solamente una parte del rendimiento y aprendizaje obtenido por los estudiantes, pero dan una luz para poder mejorar el curso en el siguiente cuatrimestre.

\section{METODOLOGÍA}

Para todas las pruebas realizadas, se utilizó una muestra de 47 estudiantes para trabajar el estudio, lo cual fue la matrícula del curso Manejo poscosecha I durante el I cuatrimestre del 2012.
1. Efecto que genera la situación particular de cada estudiante en el rendimiento, aprendizaje y motivación en el curso Manejo poscosecha I

Los siguientes rubros fueron utilizados para estimar la situación específica de cada estudiante del curso Manejo poscosecha I durante el I cuatrimestre del 2012. Se utilizaron métodos cualitativos de averiguación por teléfono:

\section{(a) Consideración del nivel de dominio que tiene cada es- tudiante sobre el uso de la plataforma virtual}

La cátedra se encargó de indagar la situación de cada estudiante en cada curso con respecto a dos temas: a) sus posibilidades de acceso a internet $y$ b) el manejo de la plataforma virtual Moodle.

Con base en los resultados obtenidos en la indagación anterior, se procedió a establecer los mecanismos que ayudaron a los estudiantes identificados, tales como más llamadas telefónicas, consultas personalizadas, consultas por correo electrónico, entrega de trabajos en papel, etc.

Posteriormente, se determinó si este seguimiento tuvo algún efecto positivo mediante el análisis de la sección 1 (sobre metacognición y autorregulación) de la encuesta aplicada a los estudiantes sobre su motivación (Apéndice 1).

Esta encuesta fue un cuestionario que evaluó los siguientes rubros:

- Metacognición y autorregulación

- Material de estudio

- Metodología de trabajo

- Aprendizaje

- Evaluación

- Uso y actividades de la plataforma virtual

Las preguntas fueron tomadas de la experiencia de la cátedra (estudio en prensa sobre el curso "Control de calidad") y a partir de estudios de diversos autores que recomendaron indicadores y preguntas específicas, las cuales llevaban a la información requerida (Capacho, 2011; Cardona \& Sánchez, 2010; Gessa, 2011; Gargallo et al., 2009).

La encuesta se aplicó a todos los y las estudiantes del curso Manejo poscosecha I, durante el I cuatrimestre del año 2012, por medio de la plataforma virtual Moodle.

\section{(b) Circunstancias especiales de los y las estudiantes y cómo solventarlas}

Se determinaron las circunstancias especiales de los y las estudiantes tales como tener problemas con el trabajo, con la distancia, no tener electricidad, no tener teléfono o cualquier otro inconveniente, para ver cómo se les ayuda 
para que puedan rendir en el curso. Esto se determinó mediante llamadas telefónicas a cada uno de los estudiantes al inicio del cuatrimestre.

\section{Influencia que ejercen el Foro 1 y el I examen ordinario en la motivación, aprendizaje y rendimiento de los estudiantes del curso Manejo poscosecha I}

De las actividades analizadas en el curso, se determinó cuáles tenían un mejor resultado en la motivación, aprendizaje y rendimiento de los estudiantes.

Para realizar esta evaluación, se procedió de la siguiente manera:

a) Se evaluó la percepción de los estudiantes sobre el curso utilizando las secciones 2 hasta la 6 de la encuesta utilizada en el punto 1 (Apéndice 1).

b) Se evaluó el nivel de aprendizaje de los estudiantes basándose en la lista de chequeo que se muestra en el Apéndice 2. La manera en la que se aplicó esta lista de chequeo para cada instrumento de evaluación se explica a continuación:

i. Foro 1: se analizaron las participaciones en el foro de cada estudiante y se determinó si el estudiante cumplía con lo siguiente: i. conceptos básicos, ii. aplicación de conceptos básicos, iii. nivel de criticidad y iv. nivel de aprendizaje adquirido.

ii. Primer examen ordinario: se analizaron las respuestas de los estudiantes en cada sección del examen. El examen constó de una primera parte de respuesta breve en la que se evaluó la adquisición de conceptos básicos y una segunda parte de desarrollo en la que se analizó la aplicación de esos conceptos, la capacidad de análisis y basado en ambas partes se evaluó el nivel de aprendizaje adquirido.

Además, se evaluó el rendimiento de los estudiantes mediante el análisis de las notas finales obtenidas en cada instrumento de evaluación mencionado en b) y el ajuste de estas notas a la escala del Apéndice 2 .

\section{Análisis estadístico}

Debido a que solamente 5 de los 30 estudiantes respondieron la encuesta sobre motivación, no se realizó análisis estadístico con esta información, sino que se presenta a manera informativa en el estudio.

Se analizaron los resultados mediante una prueba de correlación de Pearson para determinar si existe relación entre las notas adquiridas por todos los estudiantes (analizado en la sección c) del punto 2) y su nivel de aprendizaje evaluado de forma cualitativa en la sección b) del punto 2 .
Además, se realizó un análisis de varianza para determinar si existen diferencias significativas entre las notas adquiridas y su nivel de aprendizaje.

\section{Elaboración de un plan de mejora para el curso Manejo poscosecha I}

Basado en los resultados obtenidos en los puntos 1, 2 y 3; la cátedra elaboró un plan de mejora que será aplicado y evaluado en el I cuatrimestre del 2013.

\section{RESULTADOS}

1. Efecto que genera la situación particular de cada estudiante en su rendimiento, aprendizaje y motivación

(a) Consideración del nivel de dominio que tiene cada estudiante sobre el uso de la plataforma virtual

Se contactó de forma telefónica a los 47 los estudiantes del curso desde el inicio del cuatrimestre ( 7 de febrero del 2012) y se logró completar esta etapa el día 14 de febrero del 2012.

Se encontró que los estudiantes se motivaron con esta llamada. Además, el 100\% de los estudiantes contactados tienen acceso al correo electrónico y pueden utilizar la plataforma virtual Moodle.

Un 78\% de estos estudiantes participaron en el Foro I y un $80 \%$ en el primer examen ordinario.

De las 5 personas no contactadas, se encontró que 4 de las 5 participaron tanto en el foro como en la aplicación del I examen ordinario.

\section{(b) Circunstancias especiales de los y las estudiantes y} cómo se solventaron

A través de las llamadas que se realizaron a los estudiantes, se encontraron cuatro estudiantes con los siguientes casos especiales:

i. Fallas en el servicio de internet.

ii. El estudiante nunca había utilizado la plataforma Moodle.

iii. El estudiante es una persona pensionada y no tiene mucho conocimiento sobre computación.

iv. El estudiante perdió su trabajo.

Solamente el estudiante pensionado no hizo uso de la plataforma, pero se le permitió entregar sus trabajos de forma impresa. El resto de los estudiantes lograron participar en el foro y realizaron el I examen ordinario. 
2. Influencia que ejercen el Foro 1 y el primer examen ordinario en la motivación, aprendizaje y rendimiento de los estudiantes del curso Manejo poscosecha I

(a) Percepción de los estudiantes sobre el curso (secciones 2 hasta 6 de la encuesta Apéndice 1)

Solamente 5 estudiantes contestaron la encuesta que se les mandó. Sus respuestas para las secciones mencionadas fueron las siguientes:

- 4 comentaron que les fue útil el Foro de cafetería para despejar dudas.

- 3 planifican su tiempo para estudiar.

- 1 estudiante expresó que las orientaciones de curso fueron claras.

- 1 estudiante consideró que recibió suficiente retroalimentación de las evaluaciones realizadas.

- 2 vieron el video de la charla del PAL para utilizar Moodle.

- 5 preferirían tener tutorías presenciales.

(b) Análisis del nivel de aprendizaje y rendimiento de los y las estudiantes

\section{Foro 1}

Hubo diferencias entre del nivel de aprendizaje y el rendimiento obtenido por los y las estudiantes en el Foro 1 (Fig. 1).

\section{Primer examen ordinario}

También hubo diferencias entre el nivel de conocimiento y el rendimiento adquiridos por los y las estudiantes en el primer examen ordinario (Fig. 2).

\section{Análisis estadístico}

Para analizar el Foro 1 y el primer examen ordinario, se aplicó la prueba de correlación de Pearson para determinar si existe relación entre el nivel de aprendizaje y el rendimiento de los y las estudiantes y un análisis de varianza por factor de aprendizaje. Los resultados se muestran en el Cuadro 1.

\section{Plan de mejora para el curso Manejo poscosecha I}

El Cuadro 2 muestra el plan de mejora elaborado para impartir el curso Manejo poscosecha I durante el I cuatrimestre del año 2013, basado en los hallazgos encontrados en la presente investigación.

\section{DISCUSIÓN}

La Cátedra de Agroindustria considera que la calidad de un curso está ligada en parte al servicio que ofrece a sus estudiantes y parte de este servicio es tomar en cuenta las posibilidades que este tiene y las limitantes que puede presentar a lo largo del cuatrimestre.

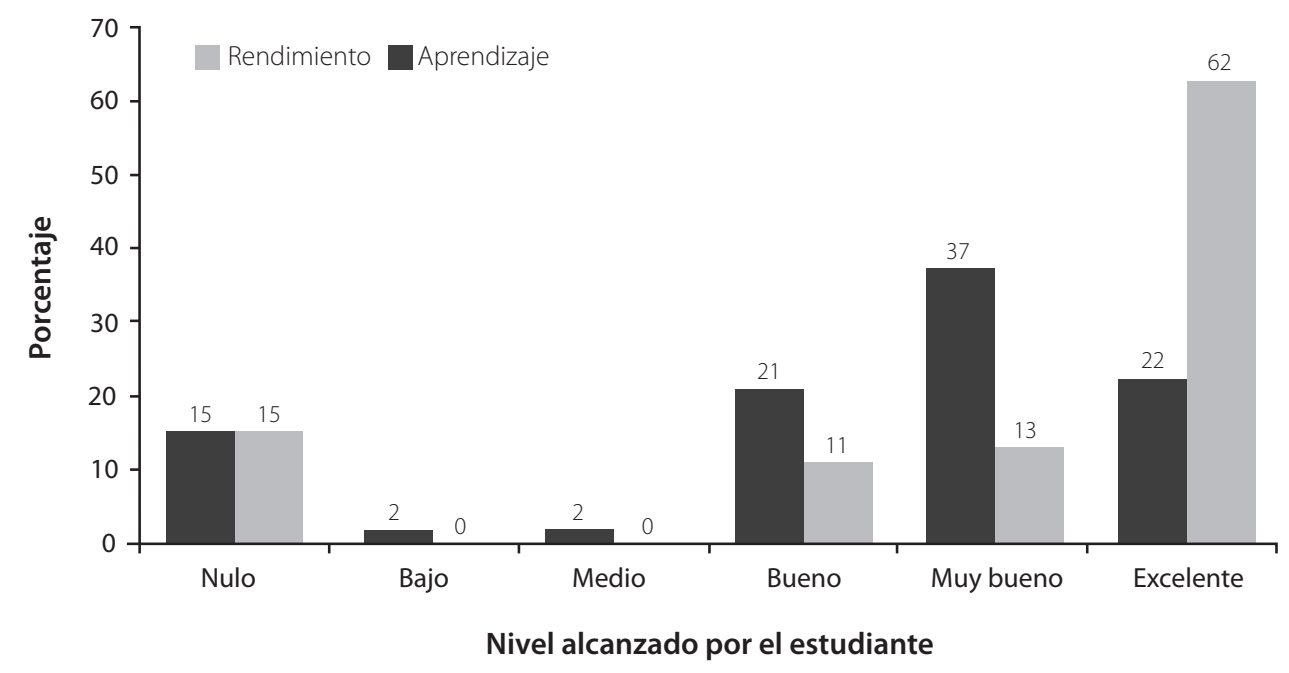

FIG. 1. Nivel de conocimiento (aprendizaje) y nota final (rendimiento) adquiridos por los y las estudiantes del curso Manejo poscosecha I en el Foro 1 por la Cátedra de Agroindustria durante el I cuatrimestre del 2012. 


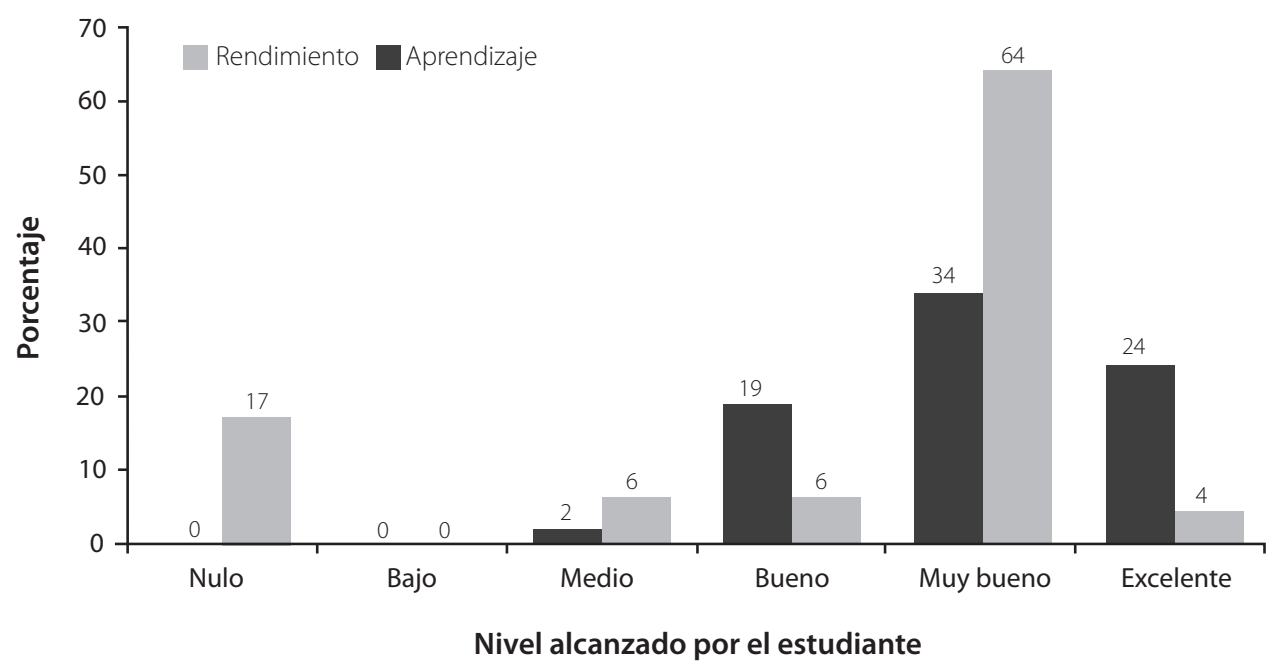

FIG. 2. Nivel de conocimiento y nota final adquiridos por los estudiantes del curso Manejo poscosecha I en el Primer examen ordinario por la Cátedra de Agroindustria durante el I cuatrimestre del 2012.

CUADRO 1

Coeficientes de correlación de Pearson y análisis de varianza por factor de aprendizaje obtenidos para determinar si existe una relación entre el nivel de aprendizaje y rendimiento adquiridos por los estudiantes del curso Manejo poscosecha I en el Foro 1 y el primer examen ordinario

\begin{tabular}{|c|c|c|c|c|}
\hline & $\begin{array}{c}\text { Conceptos básicos y } \\
\text { nota final }\end{array}$ & $\begin{array}{c}\text { Aplicación de } \\
\text { conceptos básicos y } \\
\text { nota final }\end{array}$ & $\begin{array}{l}\text { Nivel de análisis y } \\
\text { nota final }\end{array}$ & $\begin{array}{l}\text { Nivel de aprendizaje } \\
\text { adquirido y nota final }\end{array}$ \\
\hline \multicolumn{5}{|c|}{ Foro I } \\
\hline Coeficiente Pearson & 0,42 & 0,42 & 0,44 & 0,43 \\
\hline Valor $\mathrm{F}$ & 1,95 & 1,95 & 2,03 & 1,93 \\
\hline Valor $\mathrm{p}$ & 0,0699 & 0,0706 & 0,0595 & 0,0721 \\
\hline \multicolumn{5}{|c|}{ Primer examen ordinario } \\
\hline Coeficiente Pearson & 0,96 & 0,97 & 0,97 & 0,97 \\
\hline Valor F & 171,59 & 314,50 & 644,04 & 1175,88 \\
\hline Valor $\mathrm{p}$ & $<0,0001$ & $<0,0001$ & $<0,0001$ & $<0,0001$ \\
\hline
\end{tabular}


En la sección 1.a de los resultados se observa que el contactar a los y las estudiantes a inicios del cuatrimestre no sólo le aclaró a la cátedra la realidad de cada uno con respecto al uso de internet y la plataforma, sino que aumentó su motivación, ya que muchos se mostraron agradecidos por la llamada y otros evacuaron dudas que de otra forma no lo hubieran podido hacer.

El hecho de que el $100 \%$ de los y las estudiantes tuvieran acceso a correo electrónico y la plataforma virtual y que hasta un $80 \%$ hayan participado en las dos actividades evaluadas en este artículo, implica que el uso de la virtualidad en el curso Manejo poscosecha I, fue aprovechado por una cantidad importante de estudiantes, a diferencia de lo que García Sánchez, 2007 menciona en su artículo sobre las condiciones socioeconómicas de la mayoría de la población, que hacen imposible la disminución de la brecha y el incremento de la fluidez digital, por lo que esto sólo es aplicable a un reducido núcleo poblacional.

En términos de las necesidades especiales que se encontraron para cuatro estudiantes, se determinó que solamente un estudiante no pudo accesar al foro. A este estudiante se le dio la opción de entregar un ensayo escrito, pero tampoco lo entregó. Sin embargo, a pesar de lo que señala García, 2007 (citado anteriormente), la respuesta positiva del resto de los y las estudiantes que tenían problemas mostró su interés por el curso y mostró el efecto que tuvo la preocupación de la cátedra en su motivación.

Con respecto a lo anterior, el Consejo Universitario acordó indicarle a la comunidad universitaria lineamientos sobre los casos especiales de los estudiantes. Entre ellos se encuentra el fomentar el estudio y el análisis de los procesos de aprendizaje de los estudiantes en el modelo de educación a distancia tanto en la Docencia como en la Extensión, para el desarrollo de criterios objetivos de incorporación de herramientas tecnológicas y uso de plataformas virtuales (Consejo Universitario, 2012). Es por eso que este tipo de acciones mencionadas anteriormente son muy importantes en cada cátedra para fomentar la motivación, aprendizaje y buen rendimiento de los estudiantes.

Ligado a la motivación de los estudiantes, en la sección 2.a de los resultados, se muestran hallazgos muy importantes a tomar en cuenta para el siguiente cuatrimestre en que se imparta el curso. Entre éstos destaca la retroalimentación que se le debe dar a los estudiantes sobre las evaluaciones realizadas. Con respecto a esto, el CECED, 2012 comenta que el verdadero aprendizaje implica un desarrollo integral del estudiante en todas las áreas de su vida. Debe verse como un proceso que va más allá de la simple

CUADRO 2

Plan de acción a realizarse para el curso Manejo poscosecha I durante el I cuatrimestre del año 2013

\begin{tabular}{|c|c|c|c|}
\hline Hallazgo & Acción correctiva & Responsable & Fecha límite \\
\hline $\begin{array}{l}\text { Poca participación en } \\
\text { responder encuesta }\end{array}$ & $\begin{array}{l}\text { Darle un porcentaje de la } \\
\text { nota final a la encuesta, } \\
\text { autoevaluaciones y } \\
\text { coevaluaciones }\end{array}$ & Encargada de Cátedra & III cuatrimestre 2012 \\
\hline $\begin{array}{l}\text { Orientaciones de curso no } \\
\text { fueron claras para algunos } \\
\text { estudiantes }\end{array}$ & $\begin{array}{l}\text { Mejorar la redacción y } \\
\text { los lineamientos de las } \\
\text { Orientaciones }\end{array}$ & $\begin{array}{c}\text { Encargada de Cátedra y } \\
\text { Tutora }\end{array}$ & III cuatrimestre 2012 \\
\hline $\begin{array}{l}\text { No se recibió } \\
\text { retroalimentación de las } \\
\text { evaluaciones realizadas }\end{array}$ & $\begin{array}{l}\text { Diseñar actividades que } \\
\text { generen retroalimentación } \\
\text { sobre las evaluaciones }\end{array}$ & $\begin{array}{c}\text { Encargada de Cátedra y } \\
\text { Tutora }\end{array}$ & I cuatrimestre 2013 \\
\hline $\begin{array}{l}\text { Estudiantes prefieren tener } \\
\text { más tutorías presenciales }\end{array}$ & $\begin{array}{l}\text { Impartir el curso con } \\
\text { dos tutorías presenciales } \\
\text { a mitad y final de } \\
\text { cuatrimestre }\end{array}$ & Tutora & I cuatrimestre 2013 \\
\hline $\begin{array}{l}\text { Nivel de aprendizaje y } \\
\text { nota final del foro no } \\
\text { correlacionaron }\end{array}$ & $\begin{array}{l}\text { Diseñar herramientas } \\
\text { que califiquen el nivel de } \\
\text { aprendizaje y tengan un } \\
\text { porcentaje en la nota final }\end{array}$ & Encargada de Cátedra & III cuatrimestre 2012 \\
\hline
\end{tabular}


transmisión de información para lo que el estudiante construye sus propios conocimientos (CECED, 2012).

Es por lo anterior, que la cátedra debe incursionar en la evaluación alternativa para maximizar el aprendizaje de sus estudiantes, ya que a través de este tipo de evaluación se le involucra y ofrece resultados de la evaluación realizada a modo de retroalimentación, no un simple proceso de información (Cardona \& Sánchez, 2010). Junto con este proceso, se logra motivar al y a la estudiante y, según Rinaudo et al., 2003, esta motivación debe ser intrínseca, para que el y la estudiante seleccione y realice actividades por el interés, curiosidad y desafío que éstas le provocan.

Asimismo, la evaluación alternativa está relacionada con el diseño de estrategias que logren mejorar la comunicación entre estudiantes y tutores. Con respecto a esto el CECED, 2012 menciona: "el tratamiento desde el aprendizaje se refiere a la selección y diseño de las estrategias que promuevan el aprendizaje del estudiante. Si el propósito de la actividad consiste, únicamente, en un intercambio de calificaciones o una repetición de conocimientos, se pierde la intensión pedagógica del proceso formativo en línea".

Además de tener retroalimentación, los estudiantes mencionaron que prefieren tener más tutorías presenciales. A pesar de que es importante utilizar los recursos tecnológicos con los que cuenta la universidad, la presencialidad le dá seguridad a los estudiantes, ya que Silva, Guarneros et al., 2010 mencionan que la alternativa que tienen las instituciones de educación superior para superar los nuevos desafíos que plantea la sociedad del conocimiento y la interacción es recurrir a la combinación de instrucción presencial con instrucción en línea, haciendo una distinción entre lo que se aprende y se enseñan, así como a la distribución de los aprendizajes a través de distintos medios.

Para evaluar el aprendizaje y rendimiento de los estudiantes se comparó cuánto aprendieron (mediante el análisis realizado de acuerdo a la sección 2.b) y la nota final adquirida tanto en el Foro 1 como en el primer examen ordinario.

Para el Foro 1 se puede observar que la mayoría de los estudiantes obtuvieron un nivel de aprendizaje considerado como "muy bueno" en la escala utilizada, mientras que la nota final que obtuvo la mayoría fue "excelente". Al aplicar la prueba estadística de Pearson se pudo observar que la correlación entre los cuatro rubros de aprendizaje y la nota final fue baja ( $<0,44$ en promedio), y la significancia en el análisis de varianza es alta $(p=0,0699 ; p=0,0706 ; p=$ 0,0595 y $p=0,0721$ ). Por tanto, se rechaza la hipótesis nula de que el nivel de aprendizaje y la nota final sean iguales, y se concluye que lo que los estudiantes aprendieron en el Foro 1 no necesariamente se refleja en la nota final.
Recio \& Cabero, 2005, plantearon la misma hipótesis y aplicando una correlación de Pearson tampoco encontraron relación entre el enfoque de aprendizaje y el rendimiento o satisfacción de los y las estudiantes de un curso de teletransformación $(R=0,418)$.

Por otro lado, para el I examen ordinario, se puede observar que la mayoría de los estudiantes obtuvieron un nivel tanto de aprendizaje como de rendimiento, "muy bueno". Y esto se refleja en la prueba estadística de Pearson $(R=0,96-0,97)$ y en el análisis de varianza $(p<0,0001)$, dando a entender que lo que aprendieron se refleja en la nota final.

En un estudio realizado en la Universidad Politécnica de Valencia, Calduch et al., 2005, encontraron que el hecho de realizar mucha práctica para formentar el aprendizaje de la estadística, tuvo una influencia positiva en la nota final obtenida en el examen. Por otro lado, en otro estudio de la misma universidad, Gargallo et al., 2007, encontraron también correlaciones significativas entre las actitudes hacia el aprendizaje de los estudiantes de diversas disciplinas y el rendimiento académico.

En conclusión, de acuerdo al hecho de haber recibido solamente 5 respuestas de la encuesta aplicada sobre motivación, los estudiantes no responden de manera positiva en las actividades de la evaluación alternativa si éstas no tienen un porcentaje en la nota final.

Por último, se concluye que el nivel de aprendizaje que los estudiantes adquirieron con el instrumento de evaluación Foro 1 no fue el mismo que el reflejado en la nota final, mientras que sí hubo una relación clara entre el nivel de aprendizaje y el rendimiento obtenido en el I examen ordinario.

Se recomienda introducir cada vez más la evaluación alternativa en la cátedra de Agroindustria, tanto para motivar más a los estudiantes como para buscar nuevas maneras de aprender, tomando en cuenta la situación específica de cada estudiante en particular.

Además, es importante realizar modificaciones en las orientaciones de curso para que las instrucciones sean más claras para los estudiantes, así como en la retroalimentación para con las evaluaciones, ya que los estudiantes necesitan aprender de sus errores y que se les haga ver.

\section{REFERENCIAS}

Andrés, C. (2010). Efecto de tomar un curso en línea sobre el aprendizaje de los estudiantes de agroindustria en la Universidad Estatal a Distancia de Costa Rica. Cuadernos de Investigación 2, 91-108. Recuperado de http://www. uned.ac.cr/investigacion/publicaciones/cuaderno1/ documents/08-Andres-Efecto.pdf 
Andrés, C. (2012). Comunicación personal: Cátedra de Agroindustria, Escuela de Ciencias Exactas y Naturales. San José, Costa Rica: EUNED.

Borges, F. (2005). La frustración del estudiante en línea: causas y acciones preventivas. Digithum. Universitat Oberta de Catalunya. Recuperado de http://scholar.google.com/schol ar?q=Motivaci\%C3\%B3n+del+estudiante+virtual\&hl=es $\& b \operatorname{tnG}=$ Buscar\&|r=

Calduch, A; Prats, J; Giner, V y Vidal-Puig, S. (2005). Un estudio sobre la utilidad de la realización de prácticas de laboratorio en el proceso de aprendizaje y su influencia en los resultados de la evaluación del alumno. Universidad Politécnica de Valencia, Departamento de Estadística e investigación operativa aplicadas y Calidad. Recuperado de http:// bioinfo.uib.es/ joemiro/aenui/procJenui/Jen2006/ prDef0106_8e296a067a.pdf

Capacho, J. (2011). Evaluación del aprendizaje en entornos virtuales-TIC. Universidad del norte. Barranquilla, Colombia. Recuperado de http://books.google.co.cr/bo oks? id=44Q4hgDjilUC\&pg=PA $1 \& d q=$ Aprendizaje, + 2011\&hl=es\&sa $=$ X\&ei $=$ LCowT9mvBtDptge4psy5DA \&redir_esc $=y \# v=$ onepage $\& \mathrm{q}=$ Aprendizaje $\% 2 C \% 20$ $2011 \& \mathrm{f}=$ false

Cardona, D \& Sánchez, J. (2010). Indicadores básicos para evaluar el proceso de aprendizaje en estudiantes de educación a distancia en Ambiente e-learning. Formación Universitaria, 3:15-32.

CECED. (2012). Curso organización y diseño de un curso en línea. UNED, San José, Costa Rica. Recuperado de http:// campusvirtual.uned.ac.cr/lms/mod/resource/view. php? $r=37044 \&$ frameset $=$ ims\&page $=3$

Consejo Universitario. (2012). CU reflexiona sobre alternativas de acceso a la tecnología. UNED. Recuperado de http://www. uned.ac.cr/cun2/CUreflexionasobrealternativasdeaccesoalatecnologia.shtml

García-Sánchez, J. (2007). La falacia de la ampliación de la cobertura educativa mediante la utilización de las tic y la educación a distancia en la educación superior en México. Revista Iberoamericana de Educación, 45: 123-140. Recuperado de http://www.rieoei.org/rie45a05.pdf

Gargallo, B; Pérez, C; Serra, B; Sánchez, F \& Ros, I. (2007). Actitudes ante el aprendizaje y el rendimiento académico en los estudios universitarios. Revista lberoamericana de Educación, 42: 1-25. Recuperado de http://www.rieoei. org/investigacion/1537Gargallo.pdf

Gargallo, B; Suárez-Rodriguez, J \& Pérez-Perez, C. (2009). El cuestionario CEVEAPEU. Un instrumento para la evaluación de las estrategias de aprendizaje de los estudiantes universitarios. RELIEVE, 15: 1-31. Recuperado de http://www. uv.es/RELIEVE/v15n2/RELIEVEv15n2_5.htm
Gessa, A. (2011). La coevaluación como metodología complementaria de la evaluación del aprendizaje. Análisis y reflexión en las aulas universitarias. Revista de Educación, 354: 749-764

PAL-PACE-UNED. (2010). Cómo diseñar y ofertar cursos en línea: consideraciones generales. San José, Costa Rica: EUNED

Peña, C., Marzo, J., De la Rosa, J y Fabregat, R. (2002). Un sistema de tutoría inteligente adaptativo considerando estilos de aprendizaje. Universitat de Girona. Recuperado de http:// atreides.udg.edu/bcds/images/bcds/papers/pdf/un_sistema_de_tutoria_inteligente_adaptativo_considerando_estilos_de_aprendizaje.pdf

Pérez, J. 2012. Curso de Organización y Diseño de cursos en línea. Comunicación personal 7 de Marzo, 2012. CECED, San José, Costa Rica: EUNED.

Recio, M y Cabero, J. (2005). Enfoques de aprendizaje, rendimiento académico y satisfacción de los alumnos en formación en entornos virtuales. Revista de Medios y Educación, 25: 93-115. Recuperado de http://redalyc.uaemex.mx/redalyc/pdf/368/36802510.pdf

Rinaudo, M; Chiecher, A y Donolo, D. (2003). Motivación y uso de estrategias en estudiantes universitarios. Su evaluación a partir de Motivated Strategies Learning Questionnaire. Anales de Sicología, 19(1): 107-119. Recuperado de http:// digitum.um.es/xmlui/bitstream/10201/8525/1/Motivacion\%20y\%20uso\%20de\%20estrategias\%20en\%20estudiantes\%20universitarios.pdf

Sánchez-Torres, J.M. 2006. Propuesta metodológica para evaluar las políticas públicas de promoción del e-government como campo de aplicación de la Sociedad de la Información. El caso colombiano. Tesis Doctoral, Universidad Autónoma de Madrid, España.

Silva, A; Guarneros, E; Padilla, J; Varona, D y Pérez, C. (2010). La vinculación de la educación presencial y a distancia: un modelo alternativo para la educación en Latinoamérica. Revista Cognición, 24 (sin números de página). Recuperado de http://www.cognicion.net/index. php?option=com_content $\&$ view $=$ article\&id $=289$ :lavinculacin-de-la-educacin-presencial-y-a-distancia-un-modelo-alternativo-para-la-educacinsup\&catid=109: difusin-cientfica\&ltemid=215

Suárez, J y Fernández, A. 2011. Evaluación de las estrategias de autorregulación afectivo-motivacional de los estudiantes: Las EEMA-VS. Anales de Psicología, 27: 369-380. Recuperado de http://uned.academia.edu/ Jos\%C3\%A9ManuelSuarez/Papers/400065/_Suarez_ Riveiro_J.M._y_Fernandez_Suarez_A.P._2011_._Evaluacion_de_las_estrategias_de_autorregulacion_afectivomotivacional_de_los_estudiantes_Las_EEMA-VS._Anales_de_Psicologia_27_2_369-380 


\section{APÉNDICE 1}

Encuesta sobre la calidad del material utilizado en el curso Manejo poscosecha I para

los estudiantes que lo llevaron en el I cuatrimestre del 2012

\section{Universidad Estatal a Distancia \\ Escuela de Ciencias Exactas y Naturales \\ Cátedra de Agroindustria}

Encuesta sobre el curso Manejo poscosecha I

$$
\text { Código } 574
$$

Nombre:

Carrera:

Fecha:

Instrucciones generales: Conteste cada pregunta respondiendo sí o no. Existen 2 preguntas abiertas solamente.

\section{Sección 1. Metacognición y autorregulación}

a) ¿Planifica su tiempo para poder estudiar al día los temas del curso? ( ) Sí ( ) No

b) ¿Solamente estudia antes de los exámenes? ( ) Sí ( ) No

c) ¿Cómo se siente más cómodo, trabajando en grupo o individualmente? ( ) Sí ( ) No

d) ¿Le fue útil utilizar el foro de cafetería del curso para despejar dudas del curso? ( ) Sí ( ) No

e) ¿Dedico mayor tiempo para estudiar los temas más difíciles del curso? ( ) Sí ( ) No

f) ¿Elaboro esquemas, fichas o mapas mentales para estudiar? ( ) Sí ( ) No

\section{$\underline{\text { Sección 2. Material de estudio }}$}

a) ¿La unidad didáctica le fue útil para aprender los conceptos básicos del primer examen ordinario?

( ) Sí ( ) No

b) ¿El material complementario fue útil para afianzar sus conocimientos y profundizar en el tema? ( ) Sí ( ) No

c) ¿El material en general fue lo suficientemente autoexplicativo para el diseño del curso que fue un 90\% en línea? ( ) Sí ( ) No 


\section{APÉNDICE 1 (Continuación...)}

Encuesta sobre la calidad del material utilizado en el curso Manejo poscosecha I para

los estudiantes que lo llevaron en el I cuatrimestre del 2012

d) ¿Las orientaciones de curso fueron suficientemente claras para usted? ( ) Sí ( ) No

\section{Sección 3. Metodología de trabajo}

a) ¿Considero que el nivel de exigencia del curso está de acuerdo a mis posibilidades?

( ) Sí ( ) No

b) ¿Considero que la Cátedra se ha preocupado por dar un buen servicio hasta el momento?

( ) Sí ( ) No

c) ¿La tutora del curso ha dedicado el suficiente tiempo en la plataforma como para que usted pudiera evacuar sus dudas y aprender lo suficiente?

( ) Sí ( ) No

d) ¿Considera que las instrucciones que se dieron para los diferentes instrumentos de evaluación fueron claras? Justifique

( ) Sí ( ) No

e) ¿Cree que el número de tareas asignadas fue más de lo que pudo manejar?

( ) Sí ( ) No

f) ¿Considera que su tutor respondió a tiempo sus dudas y que fue abierto a recibir sus preguntas?

( ) Sí ( ) No

\section{Sección 4. Aprendizaje}

a) ¿Considero que lo que he aprendido en el curso hasta el momento es útil para aplicarlo en mi vida profesional?

( ) Sí ( ) No

b) ¿Cuál fue tema más difícil de estudiar durante el curso hasta ahora?

c) ¿Considera que la Cátedra le dio la oportunidad de aprender de manera plena a pesar de alguna dificultad que pudiera haber tenido como falta de acceso a Internet, lejanía, problemas en el trabajo? ( ) Sí ( ) No 
Encuesta sobre la calidad del material utilizado en el curso Manejo poscosecha I para

los estudiantes que lo llevaron en el I cuatrimestre del 2012

d) ¿Considero que los temas estudiados son conceptos que podría aplicar en su área de trabajo? ( ) Sí ( ) No

e) ¿Siente que desarrolló su capacidad de análisis a la hora de resolver el foro 1 y el primer examen ordinario? ( ) Sí ( ) No

\section{Sección 5. Evaluación}

a) ¿Considera que el primer examen ordinario fue elaborado de acuerdo a lo que usted estudió en la unidad didáctica? ( ) Sí ( ) No

b) ¿Considera que el foro se diseñó de acuerdo a conocimientos que usted podía manejar de acuerdo a lo estudiado y lo que podía investigar? ( ) Sí ( ) No

c) ¿Considera que se le dio suficiente retroalimentación como para corregir los errores en los que incurrió en las evaluaciones hasta el momento? ( ) Sí ( ) No.

\section{Sección 6. Uso y actividades de la plataforma virtual}

a) ¿Considera que el foro 1 fue una actividad que propició su aprendizaje? ( ) Sí ( ) No

b) ¿Considera que las autoevaluaciones propiciaron su aprendizaje en el curso?

| ( ) Sí ( ) No

c) ¿Pudo recibir o ver el video de la capacitación de cómo utilizar la plataforma Moodle por parte del PAL? ( ) Sí ( ) No

d) ¿Sus dudas con respecto al uso de la plataforma fueron aclaradas oportunamente?

( ) Sí ( ) No

e) ¿Hubiera preferido tener más tutorías presenciales? Justifique

( ) Sí ( ) No 


\section{APÉNDICE 2}

Escala para evaluar el nivel de aprendizaje adquirido por los estudiantes mediante cuatro rubros en el Foro 1 y el primer examen ordinario del curso Manejo poscosecha I durante el I cuatrimestre del 2012

\section{Escala de evaluación de los aprendizajes}

A continuación, para cada estudiante, marque con una $\mathrm{x}$ el nivel que considera tiene el estudiante con respecto al nivel alcanzado en cada uno de los rubros que se indican. El valor más alto equivale a un 5 y el más bajo a un 1 .

Nombre del estudiante:

Curso:

Cuatrimestre:

Nombre del tutor:

Foro 1

\begin{tabular}{|c|c|c|c|c|}
\hline $\begin{array}{c}\text { Conceptos } \\
\text { claros }\end{array}$ & $\begin{array}{c}\text { Aplicación de } \\
\text { conceptos }\end{array}$ & $\begin{array}{c}\text { Criticidad y } \\
\text { análisis }\end{array}$ & $\begin{array}{c}\text { Nivel de } \\
\text { aprendiazaje } \\
\text { adquirido }\end{array}$ \\
\hline
\end{tabular}

Primer examen ordinario

\begin{tabular}{|c|c|c|c|c|}
\hline $\begin{array}{c}\text { Conceptos } \\
\text { claros }\end{array}$ & $\begin{array}{c}\text { Aplicación de } \\
\text { conceptos }\end{array}$ & $\begin{array}{c}\text { Criticidad y } \\
\text { análisis }\end{array}$ & $\begin{array}{c}\text { Nivel de } \\
\text { aprendizaje } \\
\text { adquirido }\end{array}$ \\
\hline
\end{tabular}


